OPEN ACCESS

Check for updates

\title{
Sex differences in mortality among binational cohort of people with chronic kidney disease: population based data linkage study
}

\author{
Nicole L De La Mata, ${ }^{1}$ Brenda Rosales, ${ }^{1}$ Grace MacLeod, ${ }^{2,3}$ Patrick J Kelly, ${ }^{1}$ Philip Masson, ${ }^{4}$ \\ Rachael L Morton, ${ }^{5}$ Kate Wyburn, ${ }^{3,6}$ Angela C Webster ${ }^{1,7}$
}

${ }^{1}$ Sydney School of Public

Health, Faculty of Medicine and

Health, University of Sydney,

Camperdown, NSW, Australia

${ }^{2}$ School of Medicine, University of Notre Dame, Fremantle, WA,

Australia

${ }^{3}$ Sydney Medical School,

Faculty of Medicine and

Health, University of Sydney,

Camperdown, NSW, Australia

${ }^{4}$ Department of Renal Medicine,

Royal Free London NHS

Foundation Trust, London, UK

${ }^{5}$ NHMRC Clinical Trials Centre,

Faculty of Medicine and

Health, University of Sydney,

Camperdown, NSW, Australia

${ }^{6}$ Renal Department, Royal Prince Alfred Hospital, Camperdown,

NSW, Australia

${ }^{7}$ Centre for Renal and Transplant Research, Westmead Hospital, Sydney, NSW, Australia

Correspondence to:

N L De La Mata

nicole.delamata@sydney.edu.au (or @Nickii_dlm on Twitter;

ORCID 0000-0001-7739-3656)

Additional material is published online only. To view please visit

the journal online.

Cite this as: $B M J$ 2021;375:e068247

http://dx.doi.org/10.1136/

bmj-2021-068247

Accepted: 22 October 2021

\section{ABSTRACT}

OBJECTIVE

To evaluate sex differences in mortality among people with kidney failure compared with the general population.

DESIGN

Population based cohort study using data linkage.

SETTING

The Australian and New Zealand Dialysis and Transplant Registry (ANZDATA), which includes all patients receiving kidney replacement therapy in Australia (1980-2019) and New Zealand (19882019). Data were linked to national death registers to determine deaths and their causes, with additional details obtained from ANZDATA.

\section{PARTICIPANTS}

Of 82844 people with kidney failure, 33329 were female (40\%) and 49555 were male (60\%); 49376 deaths (20099 in female patients; 29277 in male patients) were recorded over a total of 536602 person years of follow-up.

\section{MAIN OUTCOME MEASURES}

Relative measures of survival, including standardised mortality ratios, relative survival, and years of life lost, using general population data to account for background mortality (adjusting for country, age, sex, and year). Estimates were stratified by dialysis modality (haemodialysis or peritoneal dialysis) and for the subpopulation of kidney transplant recipients. RESULTS

Few differences in outcomes were found between male and female patients with kidney failure. However,

\section{WHAT IS ALREADY KNOWN ON THIS TOPIC?}

Many chronic diseases have sex specific mechanisms whereby women might not develop the same symptoms as men, therefore diseases could go unrecognised leading to systematic differences in care

Chronic kidney disease is more prevalent in women, but men have a faster progression to kidney failure and represent most of the population with kidney failure Few studies have explored sex differences in survival among the population with kidney failure, with most focusing on comparisons within the population rather than expected survival seen in the general population

\section{WHAT THIS STUDY ADDS?}

The life limiting impact of kidney failure is greater for women than men; the female survival advantage in the general population is lost in the presence of kidney failure Female patients with kidney failure had more excess deaths, worse relative survival, and greater life years lost compared with male patients with kidney failure; the greatest disparity was seen in younger ages and from certain causes of death (such as cardiovascular disease)

Kidney transplantation improved survival in both sexes and reduced sex differences in excess mortality, although female patients still had more life years lost compared with the general population, female patients with kidney failure had greater excess all cause deaths than male patients (female patients: standardised mortality ratio $11.3,95 \%$ confidence interval 11.2 to 11.5, expected deaths 1781 , observed deaths 20099 ; male patients: $6.9,6.8$ to 6.9 , expected deaths 4272 , observed deaths 29 277). The greatest difference was observed among younger patients and those who died from cardiovascular disease. Relative survival was also consistently lower in female patients, with adjusted excess mortality $11 \%$ higher (95\% confidence interval $8 \%$ to $13 \%$ ). Average years of life lost was 3.6 years (95\% confidence interval 3.6 to 3.7 ) greater in female patients with kidney failure compared with male patients across all ages. No major differences were found in mortality by sex for haemodialysis or peritoneal dialysis. Kidney transplantation reduced but did not entirely remove the sex difference in excess mortality, with similar relative survival $(\mathrm{P}=0.83)$ and years of life lost difference reduced to 2.3 years ( $95 \%$ confidence interval 2.2 to 2.3 ) between female and male patients.

\section{CONCLUSIONS}

Compared with the general population, female patients had greater excess deaths, worse relative survival, and more years of life lost than male patients, however kidney transplantation reduced these differences. Future research should investigate whether systematic differences exist in access to care and possible strategies to mitigate excess mortality among female patients.

\section{Introduction}

Chronic kidney disease is defined as decreased kidney function, with an estimated glomerular filtration rate less than $60 \mathrm{~mL} / \mathrm{min} / 1.73 \mathrm{~m}^{2}$, or markers of kidney damage for at least three months. In 2017, the global prevalence of chronic kidney disease was estimated at 9.1\% (95\% confidence interval $8.5 \%$ to $9.8 \%$ ) and was ranked the 12 th leading cause of death, directly resulting in 1.2 million deaths and indirectly contributing to an additional 1.4 million deaths. ${ }^{1}$ When people have advanced chronic kidney disease, kidney failure is defined by an estimated glomerular filtration rate of less than $15 \mathrm{~mL} / \mathrm{min} / 1.73$ $\mathrm{m}^{2}$. Treatment options for kidney failure are dialysis, kidney transplantation, or palliative care. Provision of care for kidney failure accounts for around $2 \%$ of healthcare expenditure in developed countries. ${ }^{2}$ Kidney failure continues to result in premature deaths and is responsible for most of the 28.5 million years of life lost. In 2017, 35.8 million disability adjusted life years could be attributed to chronic kidney disease globally. ${ }^{1}$ 
In the general population, the life expectancy of women consistently exceeds that of men in many developed countries. This difference has been attributed to biology, such as the effect of oestrogen, ${ }^{3}$ and increased healthcare seeking behaviour and greater engagement with healthcare in women. ${ }^{4}$ However, sex inequalities in health service delivery might mean the survival advantage in women does not always persist. Growing evidence supports sex specific mechanisms whereby women might not develop the same symptoms of disease as men, leading to systematic differences in care including misdiagnosis and delays in receiving treatment. ${ }^{5}$ Women are also underrepresented in randomised clinical trials and animal models, ${ }^{6}$ which can lead to the development of drugs and treatments that are more harmful or less effective in women.

Chronic kidney disease manifests differently in men and women. Women have a higher prevalence of chronic kidney disease, but men have a faster progression to kidney failure and so represent a greater proportion of the population with kidney failure. ${ }^{7}$ ${ }^{8}$ Current evidence suggests there are differences in care received by men and women with chronic kidney disease. Women are more likely to start dialysis late at lower levels of kidney function, and have higher rates of dialysis withdrawal. ${ }^{9-12}$ After a cardiovascular event, women receiving dialysis have lower rates of cardiac catheterisation and cardiac rehabilitation. ${ }^{10}$ Serum creatinine is a commonly used indicator of kidney function, but is not directly comparable between sexes. At the same level of serum creatinine, men have better kidney function than women because of higher muscle mass on average and increased endogenous creatinine production. ${ }^{13}$ To some extent this disparity is mitigated by the use of estimated glomerular filtration rate to monitor changes in kidney function, which accounts for sex, but women might still be referred for specialist care later than men with comparable kidney function. ${ }^{14}$ These factors could contribute to the poorer long term outcomes in women with kidney failure. Recommended dialysis dosage calculations were also found to favour male body compositions in a cohort study, ${ }^{15}$ but these effects were reduced in a subsequent clinical trial. ${ }^{16}$

Systematic differences in care for kidney failure might mean that women with kidney failure have comparatively poorer survival rates compared with men. Limited studies that have explored differences in survival between men and women have mainly focused on comparisons within the population with kidney failure. These studies provide little insight into the differences in relative measures of survival that account for background mortality or expected survival outcomes in the wider population. ${ }^{17} 18$ These studies also presented only one relative measure of survival in countries without universal healthcare or were carried out in earlier eras when any health inequalities in men and women were probably amplified by other social determinants, such as socioeconomic status or ethnicity.
Our study aimed to evaluate sex differences in mortality in the Australian and New Zealand population with kidney failure by using relative measures of survival to account for expected background mortality in the general population. We hypothesised that people with kidney failure would have greater mortality than the general population, and sought to explore whether the impact of kidney failure on excess mortality was comparable between sexes.

\section{Method}

\section{Study design and setting}

We undertook a population based cohort study of all people receiving kidney replacement therapy (dialysis or kidney transplantation) in Australia (from 1 January 1980 to 31 December 2019) and New Zealand (from 1 January 1988 to 31 December 2019). Both countries have a universal healthcare system and similar population demographics. Under mandate in both countries, all deaths are captured by the Births, Deaths and Marriages Registry. Primary and secondary causes of death are reported by a medical doctor and recorded on the medical certificate. A clinician then classifies causes of death according to ICD-10 codes (international classification of diseases, 10th revision). ICD-10 is an internationally agreed and standardised coding system that is used worldwide, which enables comparisons with other populations. The primary cause of death is defined as the disease or condition that started the sequence of events that resulted in death. The secondary causes of death are defined as any other disease or condition that contributed to death.

\section{Participants, data linkage, and death outcomes} Population with kidney failure-the main study population consisted of all patients who received kidney replacement therapy as recorded on the Australian and New Zealand Dialysis and Transplant Registry (ANZDATA); a subpopulation included patients who received kidney transplantation. ANZDATA has been previously described. ${ }^{19}$ Briefly, prospective data are collected from all dialysis and transplant centres in Australia and New Zealand as they occur in real time and annually for each patient, including demographics, comorbidities, and clinical data. These data do not include people with kidney failure before they receive dialysis, those who are not receiving kidney replacement therapy, or people with kidney failure who are receiving palliative care. No data cleaning was necessary because ANZDATA conforms to an existing data dictionary.

Data linkage-all patients from ANZDATA in Australia (1980-2013) and New Zealand (1988-2017) were linked to respective national death registers to determine the date and cause of death. In Australia, probabilistic linkage to the National Death Index was undertaken by the Australia Institute of Health and Welfare, matching on personal identifiers such as date of birth, sex, and full name. In New Zealand, deterministic linkage to the Mortality Collection 
(MORT) database was undertaken by the New Zealand Ministry of Health, matching on the National Health Index number. We were restricted by the years of death data available on the national death registers at the time of linkage in 2015, which commonly have some lag time. Therefore, our analysis included cause and fact of death information for our study cohort in Australia during 1980-2013 and in New Zealand during 1988-2017. Fact of death for the remaining study cohort and follow-up (Australia during 20142019 and New Zealand during 2017-2019) was obtained from ANZDATA, which has previously been shown to have strong agreement with national death registers for fact of death, but less strongly for cause of death. ${ }^{20}$ Only deidentified patient data were made available to researchers for this study once data linkage was complete.

Death ascertainment and cause of death-ICD-10 diagnosis codes provided as the primary cause of death were used to define causes of deaths in Australia (1980-2013) and New Zealand (1980-2017). Deaths were categorised into broad causes of death, largely based on ICD chapters, and leading causes of death, ${ }^{21}$ as commonly reported in national death register reports. Appendix table 1 outlines categories for causes of death.

Time at risk was measured from the date of starting kidney replacement therapy for the kidney failure population and the earliest transplant date for the subpopulation who received kidney transplantation, until the date of death recorded on the national death register (Australia 1980-2013; New Zealand 19882017) or ANZDATA (Australia 2014-19; New Zealand: 2017-19), or 31 December 2019 (censor date), whichever occurred first. We also evaluated the effect of switching treatment modality on sex differences in mortality, considering four types of treatment: haemodialysis, peritoneal dialysis, deceased donor transplant, and living donor transplant. Patients who received haemodialysis or peritoneal dialysis were censored at first transplant date, date of death, or last known follow-up. Those who received a deceased or living donor transplant were censored at date of death or last follow-up.

As probabilistic linkage could result in a small proportion of incorrect links, ${ }^{22}$ Australian patients were censored at the date of death recorded in ANZDATA if they had died but the national death register did not capture any death. Furthermore, Australian patients with additional records after the registered date of death were considered to be alive and censored.

\section{Statistical analyses}

Patient characteristics were summarised using absolute counts and proportions, overall and stratified by sex. Causes of death during 1988-2017 were compared between sexes using logistic regression to make comparisons across age groups, trends over calendar year, and by treatment modality at death. Because of a lag in availability of Australian cause of death data, causes of death during 2013-17 were predicted with five iterations based on patient and clinical characteristics including sex, age, ethnicity, cause of kidney failure, treatment modality, ever transplanted, follow-up time, ANZDATA cause of death, and comorbidities.

We calculated crude mortality rates and estimated standardised mortality ratios by using indirect standardisation, adjusting for five year age band, sex, calendar year, and country. General population mortality rates for each country were estimated using nationwide summary level data, which provided the number of deaths given in ICD-10 codes and by five year age band, sex, and calendar year. These data are publicly available from the Australian Bureau of Statistics ${ }^{23}$ and the New Zealand Ministry of Health. ${ }^{24}$ Linear regression was used to compare rates and log transformed standardised mortality ratios between sexes, across age, and over time.

We estimated the cumulative relative survival using the life table approach and Ederer II methods, matching to the general population by country, five year age band, sex, and calendar year. ${ }^{24-26}$ The cumulative relative survival can be interpreted as the proportion of patients alive compared with the expected proportion in the general population at $x$ years of follow-up. A cumulative relative survival $<1.0$ indicates a lower proportion of the study population survived than would be expected in the matched general population. Additionally, we modelled excess mortality by using Poisson regression to estimate the excess mortality ratio. Covariates included age (categorised), sex, country, calendar year, year of follow-up, cause of kidney failure, and comorbidities (chronic lung disease, peripheral vascular disease, and coronary artery disease). An excess mortality ratio equal to 1 indicates the excess mortality (or difference in the hazard of mortality) in the study population is similar to that observed in the general population.

The life expectancy was estimated, and thereafter the expected years of life lost, as the area under the survival curve from a parametric survival model with a Gompertz distribution, stratified by sex. ${ }^{27}$ The time at risk was altered to be relative to the patient's date of birth so that the hazard of death was a function of a patient's age. Covariates, selected a priori, included year when dialysis was started or when first transplant was received, cause of kidney failure, comorbidities (chronic lung disease, coronary heart disease, peripheral heart disease, cerebrovascular disease), smoking history, ethnicity, dialysis modality (haemodialysis or peritoneal dialysis), previous dialysis months (categorised; transplant only), and country. Smoking history was not routinely collected until after 1991, and so for a small proportion of patients this information is not available. Therefore, we used multiple imputation chained equations to impute smoking history with multinomial logistic regression of five iterations. Covariates in the imputation included patient and clinical characteristics, death outcome, and follow-up time (log transformed). An additional time varying binary covariate to indicate 
the first six months of follow-up was included when modelling the life expectancy to increase the precision of survival predictions, and to adjust for differences in mortality after start of dialysis or transplantation. Life expectancies were determined by the area under the survival curve for six months in the first year and annually thereafter, from trapezoidal integration until age 110. Confidence intervals for life expectancy were determined using bootstrapping with 1000 iterations by randomly sampling the study population with replacement and refitting the Gompertz model. Average years of life lost were calculated as the difference between the estimated life expectancy in our study population and life expectancy in the general population, matching on five year age band at start of kidney replacement therapy, sex, calendar year, and country. ${ }^{2426}$ We were unable to match on ethnicity or indigenous status because general population data are not available stratified by ethnicity and so we had no comparator.

We also performed subanalyses by considering treatment modality switching for the four kidney replacement therapies (haemodialysis, peritoneal dialysis, deceased donor transplant, and living donor transplant) and the subpopulation of kidney transplant recipients overall. We used the same statistical methods as described in the main analysis to estimate mortality rates, standardised mortality ratios, and expected years of life lost, except for the inclusion of a time varying covariate to take an as-treated approach. Among patients who received dialysis, a time varying covariate for dialysis modality was included to allow patient follow-up to be split between different periods of haemodialysis or peritoneal dialysis. Similarly, in transplant recipients, a time varying covariate was included to adjust for intermittent periods of dialysis after graft failure. We stratified estimates of cumulative relative survival by initial modality because methods to include time varying covariates have not been developed yet. Data were analysed using Stata version 15 (Stata Corporation, College Station, TX, USA).

\section{Patient and public involvement}

Our study was supported by a leading national patient advocacy group, Kidney Health Australia, through a competitive funding process. We have produced an impact report including a lay person summary of our study outputs. This report has been disseminated to Kidney Health Australia for sharing among their wider patient community and audience, and is freely available on our Collaborative Centre Organ Donation Evidence website (https://organdonationevidence.org. au/celestial-study/).

\section{Results}

Sex differences within population with kidney failure

A total of 82844 patients started kidney replacement therapy in Australia (1980-2019) and New Zealand (1988-2019) and 49376 (59.6\%) died during the 536602 person years of follow-up (table 1). Seventeen patients $(<0.1 \%)$ were censored at the registered date of death because ANZDATA considered them to be alive. While over half of the patients were male (59\%), the proportions of female and male patients who died during follow-up were similar (20099 female patients (60\%); 29277 male patients (59\%)). A higher proportion of female patients were receiving peritoneal dialysis at death (5223 female patients (26\%) v 6687 male patients (23\%)), a higher proportion of male patientswere undergoing haemodialysis(12968female patients (64\%) v 19432 male patients (66\%)), however similar proportions had a functioning transplant (1908 female patients $(10 \%) v 3158$ male patients (11\%)). A higher proportion of female patients identified as Aboriginal or Torres Strait Islander (11\% v 6\%), had never smoked (61\% v 40\%), and were underweight (7\% v 3\%). Additionally, a lower proportion of female patients were of European ethnicity (58\% v 63\%), were former smokers (28\% v 46\%), overweight (26\% v $35 \%$ ), and had glomerulonephritis or immunoglobulin A nephropathy as cause of kidney failure (22\% v 27\%). Coronary heart disease $(29 \% v 37 \%)$ and peripheral vascular disease $(19 \% v 23 \%)$ also affected a lower proportion of female patients than male patients.

Haemodialysis was the most common initial kidney replacement therapy, a slightly lower proportion among female patients $(65 \% v 70 \%)$, followed by peritoneal dialysis (32\% v 27\%). Overall, about a third (22328 of $80400,28 \%$ ) switched their dialysis modality at least once; this was not substantially different between sexes (9733 of 32336 female patients (30\%) v 12595 of 48064 male patients (26\%)). Only 20\% overall (15997 of 80400 ) had a different dialysis modality at the end of the study compared with at the start of the study, and the proportion was similar for female and male patients (7027 of 32336 (21\%) v 8970 of 48064 (19\%), respectively). Switching the mode of delivery (eg, from home to dialysis centre) was more common in the 12 months before death when 31\% overall (15161 of 49376) changed dialysis modality or mode of delivery.

The subpopulation of patients who received a kidney transplant included 9269 female patients and 14847 male patients; 7668 deaths occurred (32\% in female patients and $32 \%$ in male patients) over 255233 person years. A slightly smaller proportion of female patients received a kidney transplant compared with male patients during the study (25\% v 27\%, respectively). Deceased donor transplants $(n=16898$; $70 \%$ ) were more common than living donor transplants $(n=7218 ; 30 \%)$, overall and in both sexes.

Cause of death across age groups was similar between sexes (fig 1). Across all ages, the proportion of cancer deaths was 3.3\% (95\% confidence interval $2.7 \%$ to $3.9 \%, \mathrm{P}<0.001$ ) higher in male patients; the proportion of cardiovascular deaths was also higher in male patients $(4.7 \%$ higher, $3.9 \%$ to $5.6 \%, \mathrm{P}<0.001)$. However, the proportion of deaths caused by chronic kidney disease was $6.0 \%(4.9 \%$ to $7.1 \%, \mathrm{P}<0.001)$ higher in female patients than in male patients and gastrointestinal deaths were also higher $(0.8 \%, 0.3 \%$ to $1.3 \%, \mathrm{P}=0.002$ ) across all ages. We found no difference 


\begin{tabular}{|c|c|c|c|}
\hline Characteristics & Female patients & Male patients & Total \\
\hline Total patients & $33329(40)$ & $49555(60)$ & $82884(100)$ \\
\hline Deaths during follow-up & $20099(60)$ & $29277(59)$ & $49376(60)$ \\
\hline \multicolumn{4}{|l|}{ Country } \\
\hline Australia & $27822(83)$ & $41525(84)$ & $69347(84)$ \\
\hline New Zealand & 5507 (17) & $8030(16)$ & $13537(16)$ \\
\hline \multicolumn{4}{|l|}{ Age at kidney failure, years } \\
\hline$\leq 17$ & $759(2)$ & $1037(2)$ & $1796(2)$ \\
\hline $18-34$ & $3162(9)$ & $4221(9)$ & $7383(9)$ \\
\hline $35-49$ & 6214 (19) & $8877(18)$ & $15091(18)$ \\
\hline $50-59$ & $7220(22)$ & $9989(20)$ & $17209(21)$ \\
\hline $60-79$ & $14461(43)$ & $22296(45)$ & $36757(44)$ \\
\hline$\geq 80$ & $1513(5)$ & $3135(6)$ & $4648(6)$ \\
\hline Median age, years (IQR) & $59(46-69)$ & $60(47-70)$ & $59(47-70)$ \\
\hline \multicolumn{4}{|l|}{ Year of kidney failure } \\
\hline$\leq 1995$ & $10018(30)$ & $12978(26)$ & $22996(28)$ \\
\hline $1996-2005$ & $10593(32)$ & $15739(32)$ & $26332(32)$ \\
\hline $2006-2013$ & $12718(38)$ & $20838(42)$ & $33556(40)$ \\
\hline \multicolumn{4}{|l|}{ Ethnicity* } \\
\hline European & $19419(58)$ & $31082(63)$ & $50501(61)$ \\
\hline Australian and New Zealander & $2720(8)$ & $4900(10)$ & $7620(9)$ \\
\hline Aboriginal and Torres Strait Islander & 3498 (11) & $2756(6)$ & $6254(8)$ \\
\hline Pasifika and Maori & $3743(11)$ & $4736(10)$ & 8479 (10) \\
\hline Asiant & $2818(8)$ & $3940(8)$ & $6758(8)$ \\
\hline Otherf & $1131(3)$ & $2141(4)$ & $3272(4)$ \\
\hline \multicolumn{4}{|l|}{ Cause of kidney failure } \\
\hline Diabetes & $10361(31)$ & $15434(31)$ & $25795(31)$ \\
\hline Hypertension or renal artery disease & $3262(10)$ & 6737 (14) & 9999 (12) \\
\hline Glomerulonephritis or IgA nephropathy & $7280(22)$ & $13321(27)$ & $20601(25)$ \\
\hline Polycystic kidney disease & $2548(8)$ & 3077 (6) & $5625(7)$ \\
\hline Uncertain diagnosis & $1962(6)$ & $3038(6)$ & $5000(6)$ \\
\hline Other§ & $7916(24)$ & $7948(16)$ & $15864(19)$ \\
\hline \multicolumn{4}{|l|}{ Smoking status } \\
\hline Current & 3459 (11) & $6250(14)$ & 9709 (13) \\
\hline Former & $8351(28)$ & $21195(46)$ & $29546(39)$ \\
\hline Never & $18379(61)$ & $18180(40)$ & $36559(48)$ \\
\hline Not collected & $3140(-)$ & $3930(-)$ & $7070(-)$ \\
\hline \multicolumn{4}{|l|}{ Body mass index } \\
\hline Underweight $(\leq 18.4)$ & $1965(7)$ & $1406(3)$ & $3371(5)$ \\
\hline Normal $(18.5-24.9)$ & $10432(35)$ & $15239(34)$ & $25671(35)$ \\
\hline Overweight (25.0-29.9) & $7684(26)$ & $15523(35)$ & $23207(31)$ \\
\hline Obese $(\geq 30.0)$ & $9406(32)$ & $12620(28)$ & $22026(30)$ \\
\hline Not collected & $3842(-)$ & $4767(-)$ & $8609(-)$ \\
\hline \multicolumn{4}{|l|}{ Comorbidity at kidney failure } \\
\hline Lung disease & 4535 (14) & 7299 (15) & $11834(14)$ \\
\hline Coronary heart disease & $9619(29)$ & $18430(37)$ & $28049(34)$ \\
\hline Peripheral vascular disease & 6270 (19) & $11343(23)$ & $17613(21)$ \\
\hline Cerebrovascular disease & 3847 (12) & $6282(13)$ & $10129(12)$ \\
\hline Diabetes & $13267(40)$ & $19979(40)$ & $33246(40)$ \\
\hline \multicolumn{4}{|l|}{ Initial kidney replacement therapy } \\
\hline Haemodialysis & $21660(65)$ & $34673(70)$ & $56333(68)$ \\
\hline Peritoneal dialysis & $10676(32)$ & $13391(27)$ & $24067(29)$ \\
\hline Pre-emptive transplant & $993(3)$ & $1491(3)$ & $2484(3)$ \\
\hline \multicolumn{4}{|l|}{ Transplant status } \\
\hline Transplanted during follow-up & $8276(25)$ & $13356(27)$ & $21632(26)$ \\
\hline No transplant & $24060(72)$ & $34708(70)$ & $58768(71)$ \\
\hline \multicolumn{4}{|c|}{ Previous dialysis duration before first transplant } \\
\hline 0 to $<6$ months & $1976(21)$ & $3115(21)$ & $5091(21)$ \\
\hline$\geq 6$ months to $<1$ year & $1278(14)$ & $2100(14)$ & $3378(14)$ \\
\hline$\geq 1$ to $<2$ years & $2001(22)$ & $3314(22)$ & $5315(22)$ \\
\hline$\geq 2$ to $<3$ years & $1278(14)$ & $2181(15)$ & 3459 (14) \\
\hline 23 years & $2736(30)$ & $4137(28)$ & $6873(29)$ \\
\hline Median in months (IQR) & $20(8-41)$ & $19(8-39)$ & $19(8-40)$ \\
\hline \multicolumn{4}{|c|}{$\begin{array}{l}\text { Ig=immunoglobulin G; IQR=interquartile range. } \\
\text { *Categorised based on the Australian Standard Classification of Cultural and Ethnic Groups } 2016 . \\
\text { IIncludes North-East Asian (eg, Chinese, Japanese, Korean, Taiwanese, and Mongolian), South-East Asian (eg, Burmese, Cambodian, Filipino, Indonesian } \\
\text { Lao, Malay, Singaporean, Thailander, Timorese, and Vietnamese), and Southern Asian (eg, Indian, Nepalese, Sri Lankan, and Pakistani). } \\
\text { fIncludes African, Middle East, North American and South American. } \\
\text { \$Most common other causes of kidney failure included congenital abnormalities of the kidney and urinary tract (25\%); drug and heavy metal toxicity } \\
\text { (28\%); obstructions (10\%); and cancer (4\%). } \\
\text { qData not routinely collected until after } 1991 .\end{array}$} \\
\hline
\end{tabular}


between sexes across age for respiratory $(\mathrm{P}=0.62)$ and diabetes deaths $(\mathrm{P}=0.11)$. Additionally, there was no interaction between age and sex $(\mathrm{P}>0.001)$, indicating a consistent difference in the proportion attributed to each cause of death depending on sex and age (age did not modify the association). The leading causes of death were also similar between sexes overall (appendix table 3) and by age (appendix table 4).

We found some variations in causes of death for different treatment modalities at death; however, differences between sexes were consistent with the overall findings by age group (appendix fig 1; appendix table 2). The proportion of cancer deaths was $11.3 \%$ (95\% confidence interval $10.2 \%$ to $12.4 \%$ ) lower for patients undergoing haemodialysis and 14.6\% (13.4\% to $15.7 \%$ ) lower for those receiving peritoneal dialysis compared with those who had transplantation in both sexes and across ages $(\mathrm{P}<0.001)$. The proportion of cancer deaths in patients who received living donor transplants was $6.1 \%$ (3.1\% to $9.0 \%$ ) higher compared with those who received deceased donor transplants. The proportion of deaths from chronic kidney disease in patients who received haemodialysis and peritoneal dialysis was $3.1 \%$ (1.5\% to $4.7 \%$ ) higher and $7.3 \%$ (5.5\% to $9.0 \%)$ higher, respectively, compared with those who had transplantation, in both sexes and across ages. The proportion of deaths from diabetes in patients who received haemodialysis and peritoneal dialysis was $8.1 \%$ (7.4\% to $8.7 \%$ ) higher and $10.7 \%$
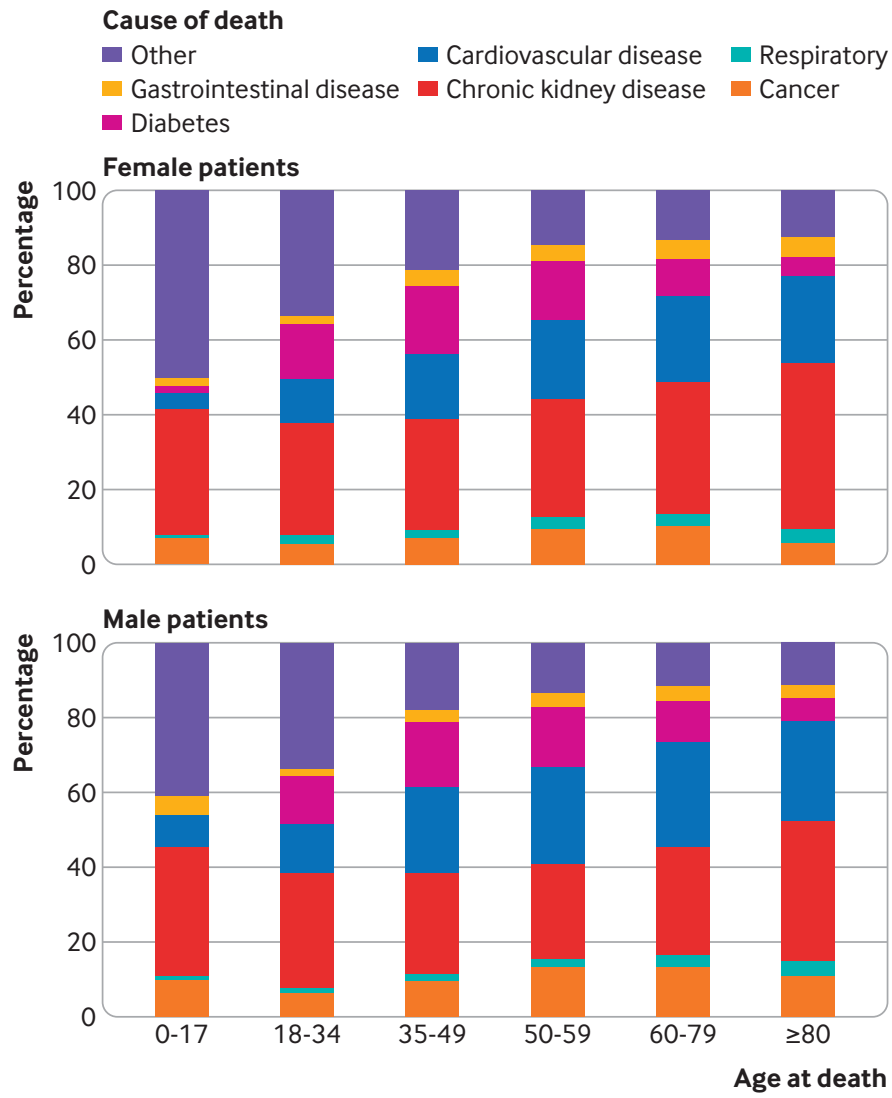

Fig 1 | Leading causes of death in patients receiving kidney replacement therapy in Australia and New Zealand by sex and age at death. See appendix table 1 for other causes of death by sex and appendix table 2 for absolute counts by sex
(9.9\% to $11.6 \%$ ) higher compared with transplant recipients, respectively, in both sexes and across ages $(\mathrm{P}<0.001)$. The proportion of cardiovascular deaths was $5.1 \%$ (2.3\% to $7.9 \%)$ lower for patients who received living donor transplants compared with those who received deceased donor transplants. No other significant differences were found for causes of death in patients who received deceased donor and living donor transplants.

Over time, male patients had a 3.1\% (95\% confidence interval $2.5 \%$ to $3.7 \%$ ) higher proportion of cancer deaths and $5.2 \%$ (4.3\% to $6.0 \%$ ) higher proportion of cardiovascular deaths compared with female patients $(\mathrm{P}<0.001$; fig 2). Female patients had a $5.4 \%$ (4.4\% to $6.6 \%$ ) higher proportion of deaths from chronic kidney disease and a $0.9 \%(0.4 \%$ to $1.4 \%)$ higher proportion of deaths from gastrointestinal disease compared with male patients $(\mathrm{P} \leq 0.001)$. No difference between sexes was found over time for respiratory deaths $(\mathrm{P}=0.93)$ and diabetes deaths $(\mathrm{P}=0.36)$. Additionally, no significant interactions were found in causes of death over time between sexes $(\mathrm{P}>0.1)$. In both sexes, cardiovascular disease deaths reduced the most over calendar time by $7.0 \%(5.4 \%$ to $8.7 \%$; $\mathrm{P}<0.001)$ and cancer deaths increased the most by $2.6 \%$ (1.6\% to $3.6 \%$; $\mathrm{P}<0.001)$ in 2012-17 compared with 1994-99.

\section{Sex differences compared with general population}

Overall, mortality rates were similar between sexes in patients with kidney failure across age (fig 3 upper panel; $\mathrm{P}>0.80$ ) and by treatment modality at death (appendix fig 2; P>0.80). However, compared with the general population, the number of excess deaths in female patients with kidney failure was much greater than that in male patients with kidney failure (fig 3 lower panel; sex interaction $\mathrm{P}<0.001$ ). This finding was most evident in younger patients; in female patients aged 18-34 years with kidney failure, the standardised mortality ratio was 53.6 (95\% confidence interval 49.2 to 58.4) and in male patients it was 16.7 (15.4 to 18.2). The excess death rate in female patients with kidney failure was also greater across calendar years (fig 4 upper panel). However, this figure has decreased over time at a greater rate in female patients than in male patients (sex interaction $\mathrm{P}<0.001$ ), but has stagnated in recent years. In female patients, the standardised mortality ratio reduced from 17.0 (14.5 to 19.9) in 1988 to 9.6 (9.0 to 10.2 ) in 2019; in male patients the ratio reduced from 8.1 ( 7.0 to 9.4 ) in 1988 to 6.4 (6.1 to 6.7 ) in 2019. The greater excess deaths in younger female patients and trends in sex disparity over time were consistent for different dialysis modalities and transplant types (appendix fig 3).

Overall, female patients with kidney failure had greater excess all cause deaths compared with male patients (standardised mortality ratio 11.3, 11.2 to 11.5 , and $6.9,6.8$ to 6.9 , respectively), but excess deaths did vary by cause (fig 4 lower panel). The greatest sex disparity was for deaths from cardiovascular disease (standardised mortality ratio 8.7, 8.4 to 9.0, and 5.7, 5.6 to 5.9, for female and male 

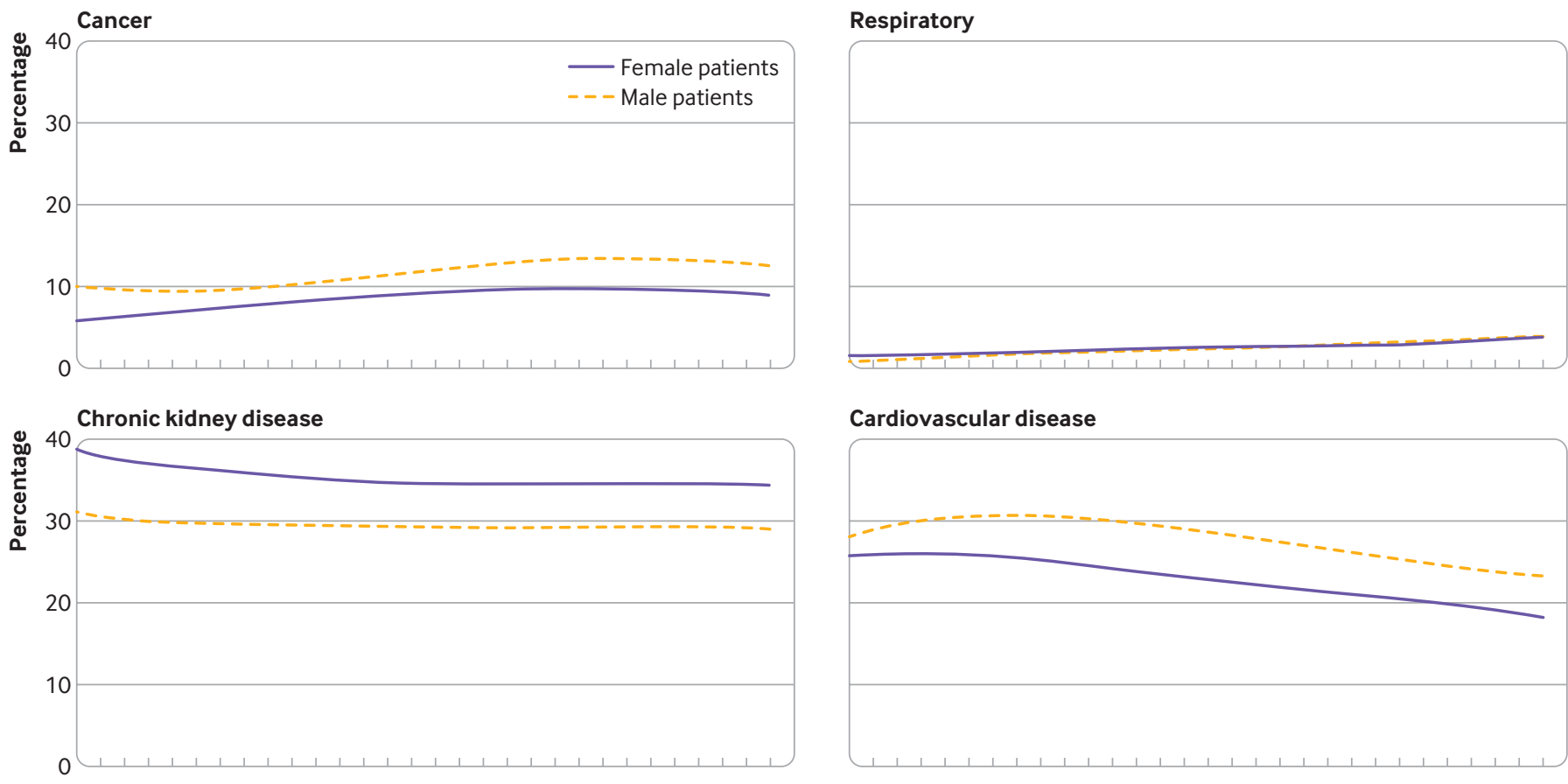

Cardiovascular disease

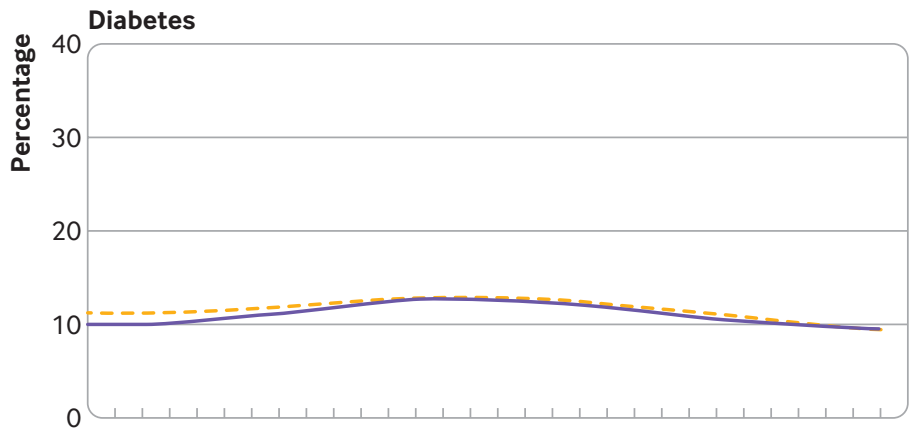

Gastrointestinal disease
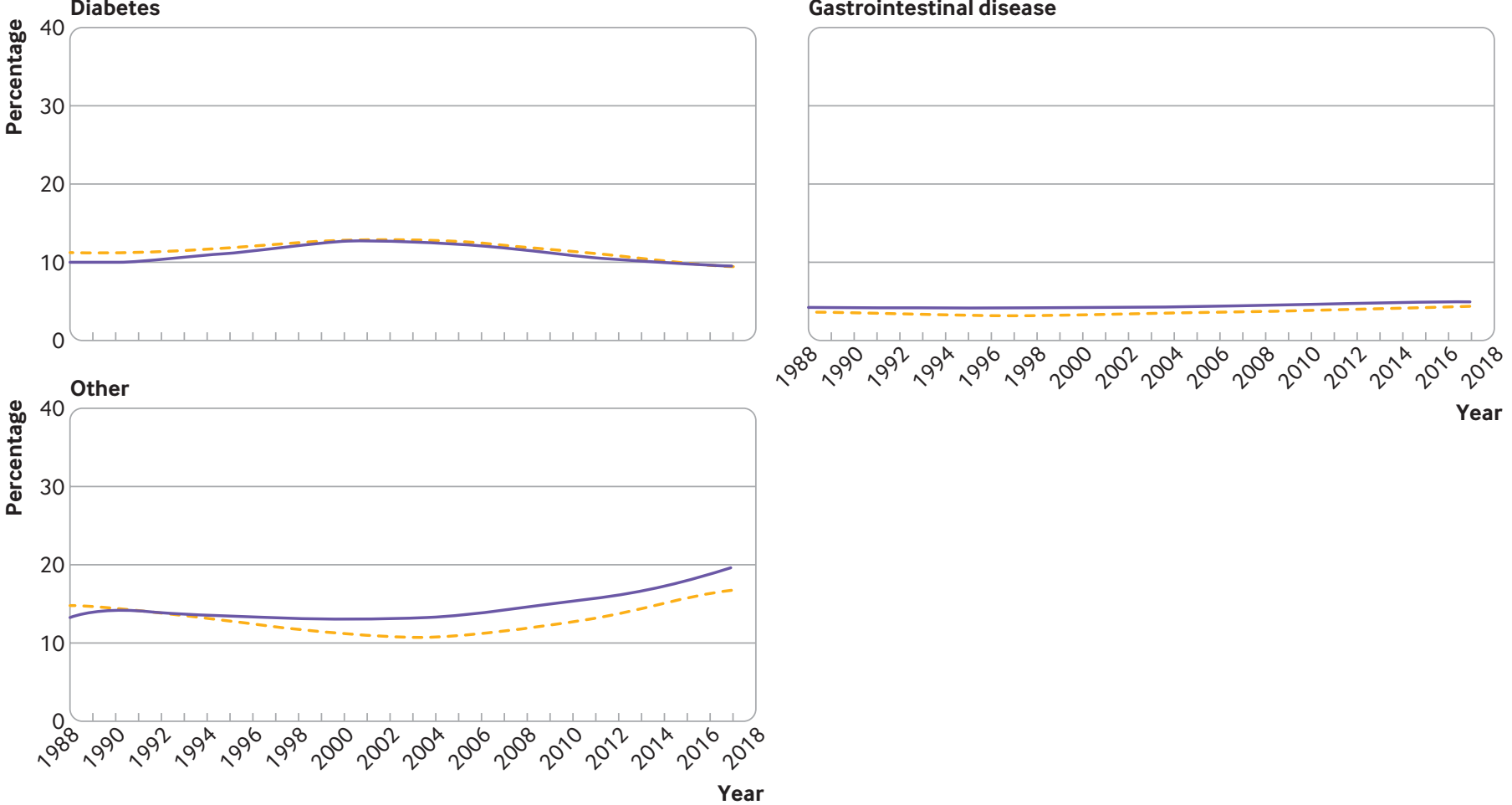

Fig 2 | Percentage of deaths by cause in patients receiving kidney replacement therapy in Australia and New Zealand over time, 1988 -2017 (Lowess lines are used). See appendix table 1 for other causes of death by sex and appendix table 2 for absolute counts by sex

patients, respectively) and gastrointestinal disease $(15.1,13.9$ to 16.3 , and $7.3,6.8$ to 7.9 , for female and male patients, respectively).

Survival rates among male patients with kidney failure compared with the general population were consistently better than the corresponding figures for female patients (fig 5 upper panel). At one year, the relative survival rate in female patients was $0.82(95 \%$ confidence interval 0.81 to 0.83 ) and in male patients it was 0.83 (0.83 to 0.84$)$. These rates decreased to 0.39 (0.38 to 0.39 ) and 0.41 ( 0.40 to 0.42 ), respectively, at 10 years. At any given time, the adjusted excess mortality was $11 \%$ higher among female patients with kidney failure compared with male patients with kidney failure (adjusted excess mortality ratio 1.11, 95\% confidence interval 1.08 to $1.13, \mathrm{P}<0.001$ ); however, this ratio differed depending on treatment modality. Among those initially receiving haemodialysis, female 

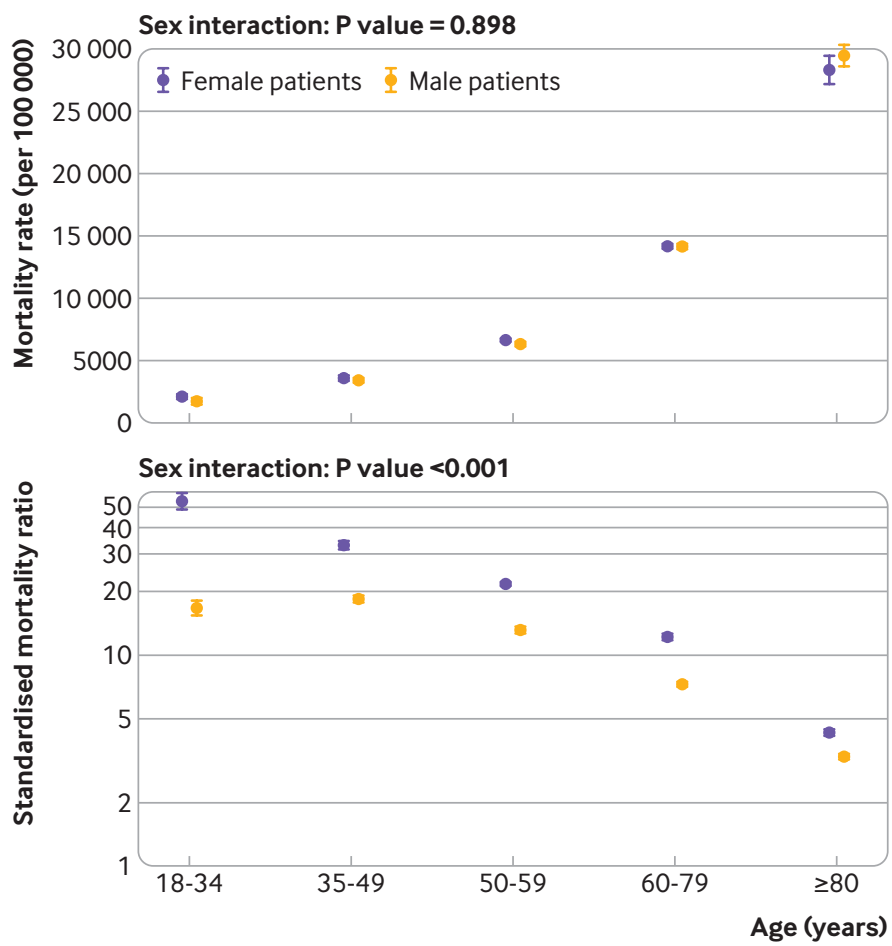

Fig 3 | Estimates of mortality rates by age and sex (upper panel) and standardised mortality ratios by age and sex (lower panel) for patients receiving kidney replacement therapy in Australia and New Zealand

patients had an excess mortality $7 \%$ higher than male patients (adjusted excess mortality ratio $1.07,1.04$ to $1.10, \mathrm{P}<0.001)$. In patients initially receiving peritoneal dialysis, the sex difference in excess mortality varied significantly by age: female patients aged 30-49 years had 15\% lower excess mortality compared with male patients $(0.85,0.76$ to $0.95, \mathrm{P}=0.004)$. Female patients aged $\leq 29$ years $(\mathrm{P}=0.48)$ and $50-64$ years $(\mathrm{P}=0.42)$ had no excess mortality compared with male patients. However, female patients aged 65-74 years had 8\% excess mortality $(1.08,1.01$ to $1.15, \mathrm{P}=0.034)$ and those aged $\geq 75$ years had $24 \%$ excess mortality (1.24, 1.11 to $1.38, \mathrm{P}<0.001)$ compared with male patients. This sex difference in relative survival was not seen in those who received a kidney transplant (fig 5 lower panel; $1.01,0.95$ to $1.06, \mathrm{P}=0.83$ ), regardless of whether they received a deceased donor transplant (0.98, 0.92 to $1.03, \mathrm{P}=0.41$ ) or living donor transplant (1.10, 0.95 to $1.27, \mathrm{P}=0.20$ ).

The average years of life lost in female patients with kidney failure was 3.6 years (95\% confidence interval 3.6 to 3.7) greater than in male patients with kidney failure $(\mathrm{P}<0.001$; fig 6 upper panel), adjusting for age. In those aged 15 years at the start of kidney replacement therapy, female patients had 33.2 years of life lost (95\% confidence interval 32.4 .5 to 34.0) while male patients had 27.7 years of life lost (27.0 to 28.5). The average years of life lost decreased to 17.2 years (17.1 to 17.4$)$ in female patients and 14.3 years (14.1 to 14.4 ) in male patients aged 60 years at the start of kidney replacement therapy. The average years of life lost for each dialysis modality was also higher among female patients. The sex difference was consistent between dialysis modalities (haemodialysis
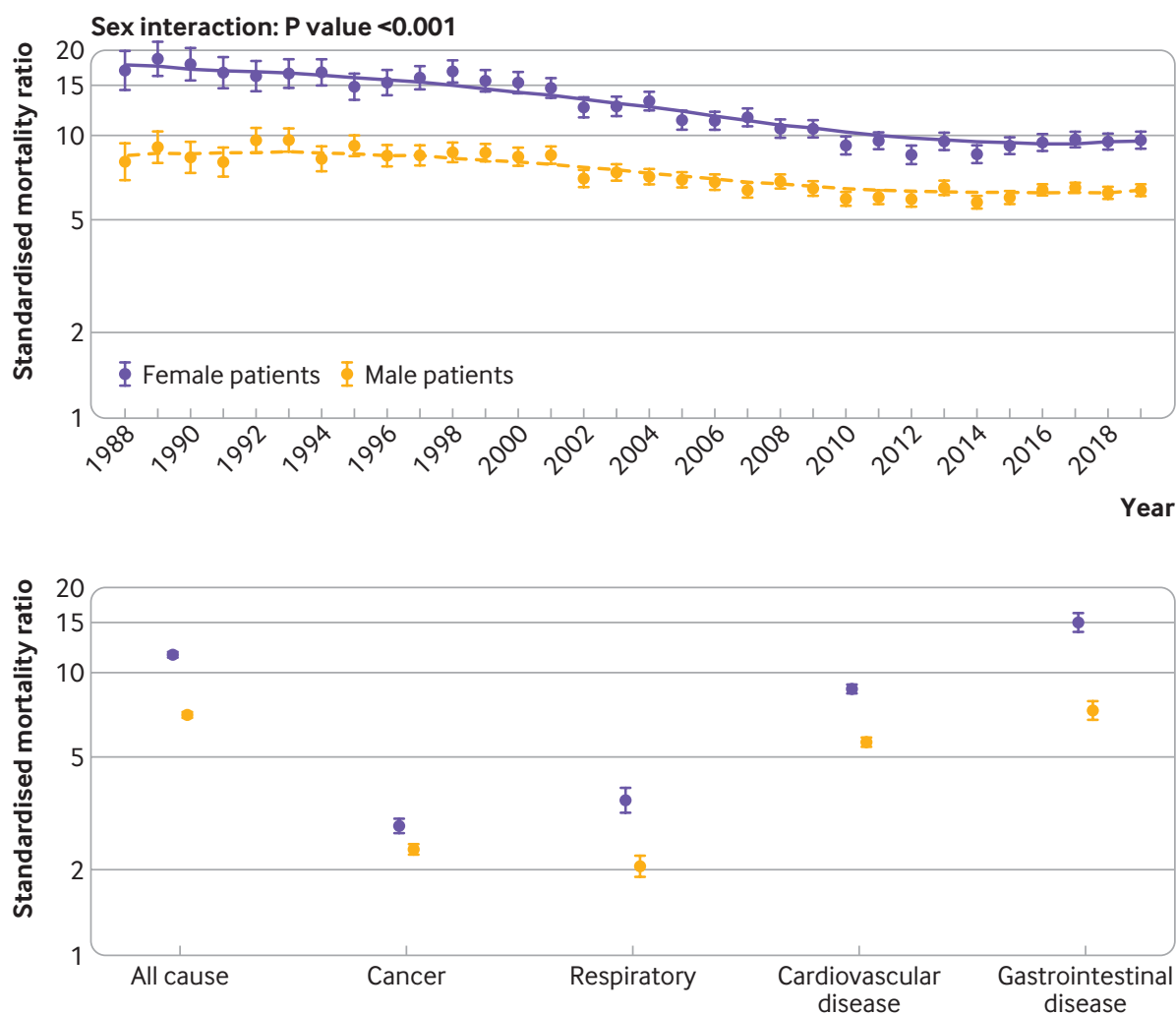

Fig 4 | Standardised mortality ratios by calendar year (with Lowess fitted line; upper panel) and by cause of death (lower panel) for patients receiving kidney replacement therapy in Australia and New Zealand 

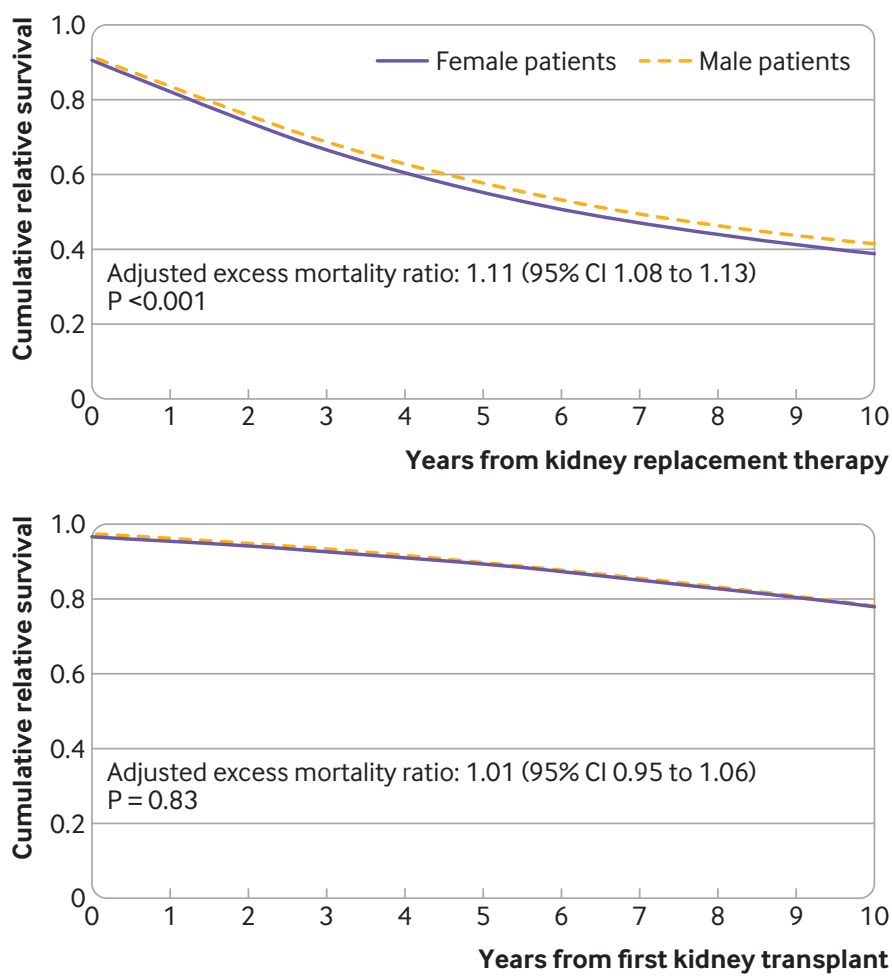

Fig 5 | Survival in population receiving kidney replacement therapy relative to matched general population (relative survival) in Australia and New Zealand by sex and time since start of kidney replacement therapy (upper panel) and first kidney transplant (lower panel)

or peritoneal dialysis); on average, female patients lost 3.4 years of life (3.3 to 3.5) more than their male counterparts (appendix fig 4). This sex difference was
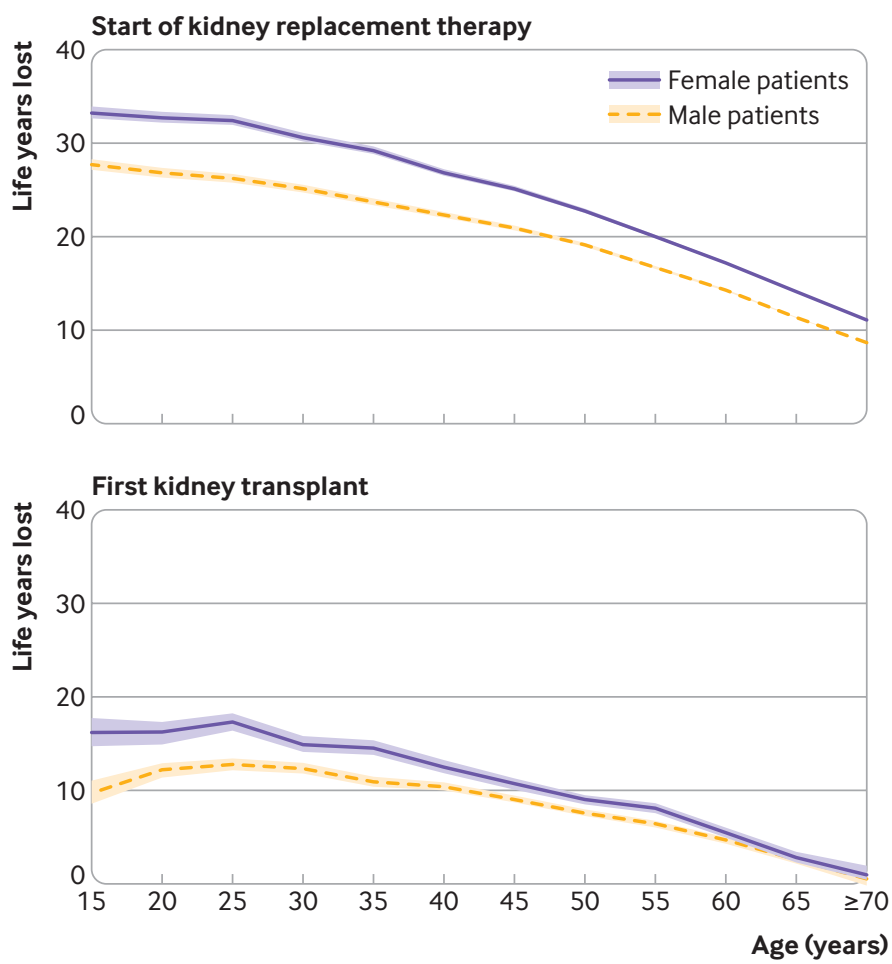

Fig 6 | Expected life years lost by sex and age at start of kidney replacement therapy (upper panel) and first kidney transplant (lower panel). Age represents five year age band except where indicated otherwise reduced in those who received a kidney transplant, in whom the average years of life lost was 2.3 years (2.2 to 2.4) greater in female patients compared with male patients (fig 6 lower panel), adjusting for age $(\mathrm{P}<0.001)$. The sex difference in average years of life lost was consistent regardless of type of donor transplant (deceased donor: average years of life lost in female $v$ male patients 2.2, confidence interval 2.1 to 2.3; living donor transplant 2.8, 2.7 to 2.9). However, the average years of life lost in deceased donor transplants was 8.4 years (8.3 to 8.5) more than living donor transplants. Appendix tables 5 and 6 present life expectancy and years of life lost estimates.

\section{Discussion \\ Principal findings}

In this binational population based study, we found that the life limiting impact of kidney failure is greater for women than men, with the female survival advantage in the general population entirely lost in the presence of kidney failure. Sex differences in mortality were only apparent when compared with the general population. Female patients with kidney failure experienced more excess deaths, worse relative survival, and greater life years lost compared with male patients with kidney failure. The greatest sex disparity in excess deaths was seen in younger ages and from certain causes of death. We observed some differences between sexes in causes of death: more cancer and cardiovascular deaths were found for male patients, while female patients had more excess deaths from cardiovascular disease and gastrointestinal disease. Improvements were found over calendar time, with a substantial reduction in female excess mortality; however, the rate has stabilised in recent years and not approached male estimates. Kidney transplantation also improved survival and lessened sex differences in excess mortality, although female patients still had more years of life lost. We found no major differences in female excess mortality between those on haemodialysis or peritoneal dialysis, or between those who received deceased or living donor transplant.

\section{Comparison with other studies}

Our findings show that female patients with kidney failure had comparably poorer survival than male patients with kidney failure. Overall we found that female patients with kidney failure had 11\% higher excess mortality at any given time, and on average had four more years of life lost than their male counterparts. As such, life expectancies in female patients with kidney failure were consistently lower than in male patients with kidney failure. The higher excess mortality among female patients was also observed for those receiving haemodialysis ( $7 \%$ excess mortality), but among those receiving peritoneal dialysis the higher rate was only present in those aged $\geq 65$ years ( $>8 \%$ excess mortality). Few studies have explored sex differences after kidney failure by comparing outcomes with expected outcomes in the general population ${ }^{17}{ }^{18}$; none of these studies was based in countries with universal healthcare 
coverage. The sex disparity in excess mortality seen in our study was greater than previous reports in the general population for cardiovascular disease ${ }^{28} 29$ and cancer. ${ }^{30}$ After an acute myocardial infarction, women experienced a $7 \%$ higher excess mortality ${ }^{28}$ and had an average 1.9 years of life lost more than men. ${ }^{29}$ However, in patients with cancer, the average years of life lost was 2.2 years greater in women than men $(14.9 v 12.7$ years, respectively). ${ }^{30}$ Findings from a cohort with kidney failure in the United States showed a higher excess mortality than our study at $18 \%$, but a similar average years of life lost (3.2 years greater in female patients than in male patients). ${ }^{17}$ Our life expectancy estimates are slightly higher than those reported in the US Renal Data System annual data report 2020, but depict similar trends with female patients receiving dialysis having lower life years remaining and higher years of life lost compared with male patients. ${ }^{31}$ Reasons for comparably worse survival outcomes in female patients with kidney failure might be attributed to early onset of menopause which reduces the protective effect of oestrogen, ${ }^{32} 33$ and possible differences in the delivery and quality of healthcare overall and for kidney failure. ${ }^{7}$ While receiving dialysis, women report higher symptom burden and severity, and lower quality of life compared with men. In our study cohort, there was also a higher proportion of female patients who identified as Aboriginal or Torres Strait Islander, with disadvantages that are well recognised-greater risk of death and poorer access to healthcare. ${ }^{3435}$ Aboriginal and Torres Strait Islanders represented $<10 \%$ of the kidney failure population overall, but might contribute to some of the excess mortality among female patients with kidney failure. Other reasons for poorer survival among female patients with kidney failure might include poorer access to cardiovascular health services, ${ }^{10}$ non-adherence to drugs, and lower likelihood of receiving care according to recommended guidelines. ${ }^{7}$

Sex differences in excess mortality were amplified in those who were younger and who died from certain causes, including cardiovascular disease and gastrointestinal disease. Young female patients with kidney failure had the greatest excess mortality compared with expected deaths in the general population with 54 times the excess deaths, while young male patients with kidney failure had only 17 times the excess deaths. These data could reflect a systematic difference in symptom recognition of disease based on sex, with symptoms perceived as typical of disease actually being experienced more often by male patients, but not necessarily by female patients. There is probably an under recognition by providers of disease severity and mortality risk in younger female patients with kidney failure, particularly from cardiovascular disease $e^{36}$ and stroke. ${ }^{37}$ Women in the general population have worse outcomes than men after a cardiovascular event because of differences in their symptoms, presentation, and pathophysiology. ${ }^{38}$

${ }^{39}$ An Australian study found that general practitioners are more likely to perceive cardiovascular risk as lower and are less likely to prescribe combination prevention therapy in women compared with men of the same age with established cardiovascular disease. ${ }^{40}$ In the presence of kidney disease, cardiovascular disease manifests more rapidly, leading to underestimation of cardiovascular risk and lack of adherence to prevention guidelines. ${ }^{41}$ These factors could compound in young female patients with kidney failure, resulting in greater excess mortality risk.

Studies evaluating excess deaths from gastrointestinal causes in the population with kidney failure are lacking. Previous studies have not found female patients with kidney failure are at increased risk of gastrointestinal disorders compared with male patients with kidney failure, suggesting there might be sex differences in response to treatment and long term outcomes. ${ }^{42} 43$ However, the gap in excess mortality between female and male patients with kidney failure has reduced over time. A combination of factors has resulted in reduced excess mortality among female patients, such as more kidney transplants and higher graft survival. ${ }^{7}$ Excess mortality rates from cardiovascular disease and stroke have reflected similar trends, ${ }^{36} 37$ attributed to greater use of and access to cardiovascular prevention that has probably benefited the population with kidney failure. ${ }^{44}$ This observation is supported by our findings, with the proportion of deaths from cardiovascular disease reducing over time in both sexes. In recent years, the reduction in excess deaths among the population with kidney failure has stabilised, with female patients continuing to have a greater burden of excess mortality. Further research needs to explore whether improvements in patient care for kidney failure or better access to healthcare and services can help to reduce this sex disparity.

Transplantation reduced the sex differences in excess mortality among the population with kidney failure, but still resulted in more years of life lost in female patients with kidney failure compared with the general population. Equitable access to waitlists and kidney transplantation are an ongoing issue, particularly for certain ethnicities, geographical regions, and sexes. ${ }^{45}$ Sex inequalities in access to waiting lists and kidney transplantation have only been evaluated in Canada ${ }^{46}$ and the US, ${ }^{47}$ and to a lesser degree in Australia. ${ }^{48}$ In these countries, sex disparities in transplantation were evident, particularly in earlier eras when waiting list rates were $16 \%$ higher for male patients than female patients. ${ }^{47}$ This difference has reduced over time in the US, with greater access for women. ${ }^{7}$ A systematic review in 2014 found 17 studies evaluating nephrologists' preferences and attitudes towards referral, screening, and eligibility for kidney transplantation. ${ }^{45}$ Only two studies evaluated the impact of participant sex, in which women were more likely to be considered unsuitable for transplantation compared with men of the same age and with similar comorbid conditions. Therefore, it is possible that access to transplantation is biased towards the selection of healthier women, which corresponds to our study findings of no excess mortality and comparable survival in women with kidney transplants and women in the general population. 
However, further studies evaluating access to waiting lists, remaining on waiting lists, and receiving a transplant in Australia, New Zealand, and other countries are still needed given healthcare systems are vastly different. Our study found that in patients who had received a transplantation, survival was similar between sexes and sex was not associated with excess mortality in kidney transplant recipients. Female and male patients who received kidney transplants had comparable life expectancies after transplant, resulting in more life years lost in female patients who had received a transplant because women in the general population have higher life expectancies than men.

\section{Strengths and limitations of this study}

The strength of our study is the large patient cohort that included the entire population receiving kidney replacement therapy in two countries, Australia and New Zealand, and findings were stratified by dialysis modality. We used the general population as the reference group in our estimates of excess mortality, matching on sex, age at kidney failure, calendar year, and country. The general population is the least biased and most representative comparison group because it encompasses the entire population and is not prone to selection bias or use of administrative data not intended to address our research question. Our findings are therefore highly generalisable to Australia and New Zealand, and other developed countries that offer universal healthcare. Furthermore, we used data linkage to determine death information until 2013 in Australia and 2017 in New Zealand, including causes of death classified to an international standard (ICD-10). We relied on the primary cause of death reported in national death registers to define causes of death, although differences exist when using primary and secondary causes of death. For example, cardiovascular deaths represented less than $40 \%$ of causes of death overall in both sexes. However, when also including secondary causes of death the proportion of cardiovascular deaths increases to $40 \%$. We believe primary cause of death is the most representative cause of death because it is difficult to distinguish the degree to which a disease or condition contributed to the death when relying on the primary and secondary causes of death. We used probabilistic data linkage for Australia, which might have led to incorrect links; however, this has been estimated to be relatively small at $<5$ per 1000 records $^{49}$ and there was high agreement for fact of death between the ANZDATA register and death registry. ${ }^{20}$ While additional deaths up to 2019 were determined from ANZDATA, this is unlikely to introduce any bias because of the strong agreement with national death registers. ${ }^{20}$ We were also limited to patient data collected on ANZDATA, which did not include use of preventative drugs that might lower mortality risks.

\section{Conclusions}

While the sex disparity has reduced over time, female patients with kidney failure continue to experience greater losses in survival compared with their male counterparts in recent years. Life expectancies in those with kidney failure contrast with trends in the general population, where female patients with kidney failure have lower life expectancies than male patients with kidney failure. However, kidney transplantation reduces these sex differences. Our findings will inform clinicians and patients with kidney failure about expected survival outcomes when starting dialysis and additional life gains once transplanted, helping in shared clinical decision making. Future research should explore possible reasons for and strategies to mitigate excess mortality among female patients with kidney failure, such as more access to preventative drugs, healthcare, and services. Additionally, evaluating sex differences in other chronic diseases and countries is needed to ensure access to healthcare and health outcomes are equitable between sexes.

The data reported here have been supplied by the Australia and New Zealand Dialysis and Transplant Registry (ANZDATA), the Australian Institute of Health and Welfare (AIHW), and the New Zealand Ministry of Health. The authors acknowledge the assistance provided by the AlHW in the data linkage process, and Kidney Health Australia for their financial support. The interpretation and reporting of these data are the responsibility of the authors and in no way should be seen as an official policy of interpretation of ANZDATA, AIHW, or the New Zealand Ministry of Health.

Contributors: ACW, PJK, and PM attracted funding for the data linkage. NLDLM, ACW, PJK, RLM, and KW contributed to the concept development. NLDLM and PJK contributed to the statistical design. GM and NLDLM performed the statistical analysis and interpretation of results. NLDLM prepared the draft manuscript. All authors commented on the draft manuscript and approved the final manuscript for submission. NLDLM and ACW are guarantors of this study. The corresponding author attests that all listed authors meet authorship criteria and that no others meeting the criteria have been omitted. Funding: The data linkage for this work was supported by Kidney Health Australia through KHA project grant PG3815.

Competing interests: All authors have completed the ICMJE uniform disclosure form at www.icmje.org/disclosure-of-interest/ and declare: support from Kidney Health Australia for the linked data used in the submitted work; no financial relationships with any organisations that might have an interest in the submitted work in the previous three years; no other relationships or activities that could appear to have influenced the submitted work

Ethical approval: This study received ethical approval from the University of Sydney (project No 2014/917) and AlHW (reference No EO2015/3/181). The New Zealand Ministry of Health did not require ethical approval (reference No 14/NTB/171).

Data sharing: No additional data available.

The lead author (NLDLM) confirms that the manuscript is an honest, accurate and transparent account of the study being reported and there are no important aspects of the study that have been omitted o any discrepancies from the study as planned have been reported and explained.

Dissemination to participants and related patient and public communities: The study was supported by a leading national patient advocacy group, Kidney Health Australia, through a competitive funding process. We have produced an impact report including a lay person summary of our study outputs. This has been disseminated to Kidney Health Australia for sharing among their wider patient community and audience, and is freely available on our Collaborative Centre Organ Donation Evidence website (https:// organdonationevidence.org.au/celestial-study/). This work has been presented at national and international conferences (Australian and New Zealand Society of Nephrology Annual Scientific Meeting 2020; European Renal Association and European Dialysis and Transplant Association Congress 2020; International Population Data Linkage Network 2020 Conference), and at the NSW Health Scientific Talent Competition 2020.

Provenance and peer review: Not commissioned; externally peer reviewed. 
This is an Open Access article distributed in accordance with the Creative Commons Attribution Non Commercial (CC BY-NC 4.0) license, which permits others to distribute, remix, adapt, build upon this work non-commercially, and license their derivative works on different terms, provided the original work is properly cited and the use is noncommercial. See: http://creativecommons.org/licenses/by-nc/4.0/.

1 GBD Chronic Kidney Disease Collaboration. Global, regional, and national burden of chronic kidney disease, 1990-2017: a systematic analysis for the Global Burden of Disease Study 2017. Lancet 2020:395:709-33, doi:10.1016/S0140-6736(20)30045-3

2 Jha V, Garcia-Garcia G, Iseki K, et al. Chronic kidney disease: global dimension and perspectives. Lancet 2013;382:260-72. doi:10.1016/S0140-6736(13)60687-X

3 Bush TL, Cowan LD, Barrett-Connor E, et al. Estrogen use and allcause mortality. Preliminary results from the Lipid Research Clinics Program Follow-Up Study. JAMA 1983;249:903-6. doi:10.1001/ jama.1983.03330310033022

4 O'Brien R, Hunt K, Hart G. 'It's caveman stuff, but that is to a certain extent how guys still operate': men's accounts of masculinity and help seeking. Soc Sci Med 2005;61:503-16. doi:10.1016/j. socscimed.2004.12.008

5 Sen G, Ostlin P. Gender inequity in health: why it exists and how we can change it. Glob Public Health 2008;3(Suppl 1):1-12. doi:10.1080/17441690801900795

6 Carey JL, Nader N, Chai PR, Carreiro S, Griswold MK, Boyle KL. Drugs and medical devices: adverse events and the impact on women's health. Clin Ther 2017;39:10-22. doi:10.1016/j. clinthera.2016.12.009

7 Carrero JJ, Hecking M, Chesnaye NC, Jager KJ. Sex and gender disparities in the epidemiology and outcomes of chronic kidney disease. Nat Rev Nephrol 2018;14:151-64. doi:10.1038/nrneph.2017.181

8 Cobo G, Hecking M, Port FK, et al. Sex and gender differences in chronic kidney disease: progression to end-stage renal disease and haemodialysis. Clin Sci (Lond) 2016;130:1147-63. doi:10.1042 CS20160047

9 Wright S, Klausner D, Baird B, et al. Timing of dialysis initiation. and survival in ESRD. Clin I Am Soc Nephrol 2010:5:1828-35. doi:10.2215/CJN.06230909

10 Morton RL, Schlackow I, Mihaylova B, Staplin ND, Gray A, Cass A The impact of social disadvantage in moderate-to-severe chronic kidney disease: an equity-focused systematic review. Nephrol Dial Transplant 2016;31:46-56. doi:10.1093/ndt/gfu394

11 Stel VS, Tomson C, Ansell D, et al. Level of renal function in patients starting dialysis: an ERA-EDTA Registry study. Nephrol Dial Transplant 2010;25:3315-25. doi:10.1093/ndt/gfq209

12 Obrador GT, Arora P, Kausz AT, Ruthazer R, Pereira B], Levey AS. Level of renal function at the initiation of dialysis in the U.S. endstage renal disease population. Kidney Int 1999;56:2227-35 doi:10.1038/sj.ki.4491163

13 Swedko PI, Clark HD, Paramsothy K, Akbari A. Serum creatinine is an inadequate screening test for renal failure in elderly patients. Arch Intern Med 2003;163:356-60. doi:10.1001/archinte.163.3.356

14 John R, Webb M, Young A, Stevens PE. Unreferred chronic kidney disease: a longitudinal study. Am J Kidney Dis 2004:43:825-35. doi:10.1053/j.ajkd.2003.12.046

15 Spalding EM, Chandna SM, Davenport A, Farrington K. Kt/V underestimates the hemodialysis dose in women and small men. Kidney Int 2008;74:348-55. doi:10.1038/ki.2008.185

16 Eknoyan G, Beck GJ, Cheung AK, et al, Hemodialysis (HEMO) Study Group. Effect of dialysis dose and membrane flux in maintenance hemodialysis. NEngl J Med 2002;347:2010-9. doi:10.1056/NEJMoa021583

17 van Walraven C, Manuel DG, Knoll G. Survival trends in ESRD patients compared with the general population in the United States. Am J Kidney Dis 2014:63:491-9. doi:10.1053/j.ajkd.2013.09.011

18 Villar E, Remontet L, Labeeuw M, Ecochard R. Effect of age, gender, and diabetes on excess death in end-stage renal failure. / Am SoC Nephrol 2007:18:2125-34 doi:10.1681/ASN.2006091048

19 McDonald SP, Russ GR. Australian registries-ANZDATA and ANZOD. Transplant Rev (Orlando) 2013;27:46-9. doi:10.1016/j. trre.2013.01.003

20 Sypek MP, Dansie KB, Clayton P, Webster AC, Mcdonald S. Comparison of cause of death between Australian and New Zealand Dialysis and Transplant Registry and the Australian National Death Index. Nephrology (Carlton) 2019:24:322-9 doi:10.1111/nep.13250

21 Becker R, Silvi J, Ma Fat D, L'Hours A, Laurenti R. A method for deriving leading causes of death. Bull World Health Organ 2006;84:297-304.

22 Blakely T, Salmond C. Probabilistic record linkage and a method to calculate the positive predictive value. Int J Epidemiol 2002;31:1246-52. doi:10.1093/ije/31.6.1246

23 Australian Bureau of Statistics. Life tables. 2021. https://www.abs. gov.au/statistics/people/population/life-tables.

24 Stats NZ. New Zealand cohort life tables: March 2020. 2020. https:// www.stats.govt.nz/information-releases/new-zealand-cohort-lifetables-march-2020-update.
25 Dickman PW, Coviello E. Estimating and modeling relative survival. Stata / 2015;15:186-215. doi:10.1177/1536867X1501500112

26 Australian Bureau of Statistics. Australian Historical Population Statistics, 2016. 2020. https://www.abs.gov.au/AUSSTATS/abs@.nsf/ Lookup/3105.0.65.001Main+Features12016?OpenDocument.

27 Modig K, Rau R, Ahlbom A. Life expectancy: what does it measure?BMJ Open 2020;10:e035932.

28 Alabas OA, Gale CP, Hall M, et al. Sex differences in treatments, relative survival, and excess mortality following acute myocardial infarction: national cohort study using the SWEDEHEART Registry. $\int$ Am Heart Assoc 2017;6:e007123. doi:10.1161/JAHA.117.007123

29 Bucholz EM, Normand SL, Wang Y, Ma S, Lin H, Krumholz HM. Life expectancy and years of potential life lost after acute myocardial infarction by sex and race: a cohort-based study of Medicare beneficiaries. J Am Coll Cardiol 2015;66:645-55. doi:10.1016/j. jacc.2015.06.022

30 Brustugun OT, Møller B, Helland A. Years of life lost as a measure of cancer burden on a national level. Br J Cancer 2014;111:1014-20. doi:10.1038/bjc.2014.364

31 United States Renal Data System (USRDS). USRDS Annual Data Report: Epidemiology of kidney disease in the United States. Bethesda, MD. 2020. https://adr.usrds.org/2020/end-stage-renal-disease/5-mortality

32 Vellanki K, Hou S. Menopause in CKD. Am J Kidney Dis 2018;71:7109. doi:10.1053/j.ajkd.2017.12.019

33 Cheung KL, Stefanick ML, Allison MA, et al. Menopausal symptoms in women with chronic kidney disease. Menopause 2015;22:1006-11. doi:10.1097/GME.0000000000000416

34 Gao S, Manns BJ, Culleton BF, et al, Alberta Kidney Disease Network. Prevalence of chronic kidney disease and survival among aboriginal people. J Am Soc Nephrol 2007;18:2953-9. doi:10.1681/ ASN. 2007030360

35 Hoy WE, Mott SA, Mc Donald SP. An expanded nationwide view of chronic kidney disease in Aboriginal Australians. Nephrology (Carlton) 2016;21:916-22. doi:10.1111/nep.12798

36 O'Lone E, De La Mata NL, Kelly PJ, et al. Ischaemic heart disease mortality in people with end stage kidney disease in Australia and New Zealand: a cohort study from 1980 to 2013. Nephrology (Carlton) 2017;22:47.

37 De La Mata NL, Masson P, Al-Shahi Salman R, Kelly PJ, Webster AC Death from stroke in end-stage kidney disease. Stroke 2019:50:48790. doi:10.1161/STROKEAHA.118.023644

38 Reeves MJ, Bushnell CD, Howard G, et al. Sex differences in stroke: epidemiology, clinical presentation, medical care, and outcomes. Lancet Neurol 2008;7:915-26. doi:10.1016/S1474-4422(08)70193-5

39 Polk DM, Naqvi TZ. Cardiovascular disease in women: sex differences in presentation, risk factors, and evaluation. Curr Cardiol Rep 2005;7:166-72. doi:10.1007/s11886-005-0072-9

40 Turnbull F, Arima H, Heeley E, et al. Gender disparities in the assessment and management of cardiovascular risk in primary care: the AusHEART study. Eur / Cardiovasc Prev Rehabil 2011;18:498503. doi:10.1177/1741826710389369

41 Razavian M, Heeley EL, Perkovic V, et al. Cardiovascular risk management in chronic kidney disease in general practice (the AusHEART study). Nephrol Dial Transplant 2012;27:1396-402. doi:10.1093/ndt/gfr599

42 Quraishi ER, Goel S, Gupta M. Catanzaro A, Zasuwa G, Divine G. Acute pancreatitis in patients on chronic peritoneal dialysis: an increased risk?Am J Gastroenterol 2005;100:2288-93. doi:10.1111/j.15720241.2005.41646.x

43 Anderson JE, Yim KB, Crowell MD. Prevalence of gastroesophageal reflux disease in peritoneal dialysis and hemodialysis patients. Adv Perit Dial 1999;15:75-8

44 Chow FY, Polkinghorne KR, Chadban SJ, Atkins RC, Kerr PG. Cardiovascular risk in dialysis patients: a comparison of risk factors and cardioprotective therapy between 1996 and 2001. Nephrology (Carlton) 2003:8:177-83. doi:10.1046/j.1440-1797.2003.00157.x

45 Tong A, Hanson CS, Chapman JR, et al. The preferences and perspectives of nephrologists on patients' access to kidney transplantation: a systematic review. Transplantation 2014:98:682 91. doi:10.1097/TP.0000000000000336

46 Schaubel DE, Stewart DE, Morrison HI, et al. Sex inequality in kidney transplantation rates. Arch Intern Med 2000;160:2349-54. doi:10.1001/archinte.160.15.2349

47 Wolfe RA, Ashby VB, Milford EL, et al. Differences in access to cadaveric renal transplantation in the United States. Am I Kidney Dis 2000:36:1025-33. doi:10.1053/ajkd.2000.19106

48 Sypek MP, Clayton PA, Lim W, et al. Access to waitlisting for deceased donor kidney transplantation in Australia. Nephrology (Carlton) 2019;24:758-66. doi:10.1111/nep.13484

49 Centre for Health Record Linkage. Master Linkage Key Quality Assurance. Sydney, Australia. 2012. https://www.cherel.org.au/ media/24160/qa_report_2012-a.pdf

Web appendix: Appendix figures

Web appendix: Appendix tables 